\title{
Blockchain and Government Transformation
}

\author{
Teresa Guarda $^{1,2,3,4,6(\bowtie)}(\mathbb{D})$, Maria Fernanda Augusto ${ }^{1,2,3,6}$ (D), \\ Lidice $\mathrm{Haz}^{1,3}$ (D), and José María Díaz-Nafría ${ }^{1,5,6}$ (D) \\ 1 Universidad Estatal Península de Santa Elena, La Libertad, Ecuador \\ tguarda@gmail.com,mfg.augusto@gmail.com, \\ victoria.haz@hotmail.com, jdian@unileon.es \\ ${ }^{2}$ Universidad de las Fuerzas Armadas, Salinas, Ecuador \\ ${ }^{3}$ CIST - Centro de Investigación en Sistemas y Telecomunicaciones, \\ Universidad Estatal Península de Santa Elena, La Libertad, Ecuador \\ 4 Algoritmi Centre, Minho University, Guimarães, Portugal \\ 5 Madrid Open University, Madrid, Spain \\ ${ }^{6}$ BiTrum Research Group, Madrid, Spain
}

\begin{abstract}
In today's digital age, transactions are made increasingly in the virtual universe. Some do all financial transactions online, but some do not even want to think about this possibility since the consideration is unreliable. To make online financial transactions more reliable, many technologies have already been tested. Since passwords, tokens, physical code generation accessories, there have been many attempts, being the most elaborate, the blockchain. Governments, especially in developed countries, often find it difficult to gain the trust of their citizens, especially when it comes to evidence of service provision and improvement of existing services. In developing countries, blockchain requests from governments would be useful in eliminating some important problems, such as corruption, while ensuring more effective deployment and distribution of resources. The adoption of such technologies can also help to facilitate better use of resources. In this paper, we will analyze the impact of the application of blockchain technology on e-government.
\end{abstract}

Keywords: Blockchain $\cdot$ e-Government $\cdot$ Security $\cdot$ Digital transformation $\cdot$ Competitiveness

\section{Introduction}

Information and communication technologies provide advances to society, and the role of the State tends to favor the population by offering better conditions of access to information or its services, through more interactive and participatory electronic means with the citizen, from e-Government perspective.

e-Government tends to create a new type of public service, based on an increasingly integrated and effective relationship with society, whose public institutions start to articulate public actions and policies, based on a more modernized service provision, and a an increasingly integrated database and information, in order to offer a better service to the population. 
The transformation of government services and governance can be seen as a citizen's right to request, through electronic e-government, at any time, the institution of the State and its public services, in a logic of functioning $24 \mathrm{~h}$ a day, in which the request for services and information would be made at any time or instant, via Internet, by the citizen. In this perspective, it is imperative to guarantee the security and privacy of the network, being able to be operationalized through blockchain technologies.

Blockchain is seen as a trust network, which will be executed on the internet data transfer protocols, allowing business to be carried out automatically and at a reduced cost between people, companies and countries. Every record of information or execution results must pass through the network sieve to be validated. This is what makes this technology so precious, as it ensures that all nodes in the network agree and have exactly the same information, avoiding common fraud situations in systems that depend on the validation of a third party.

It is very common to have some confusion with the terms Blockchain and Bitcoin, since Blockchain is the technological platform used for the operation of the Bitcoin network and several other cryptocurrencies; and Bitcoin is the first and most wellknown application of Blockchain technology. Blockchain technology emerged in 2009 as bitcoin infrastructure, an indisputable phenomenon, blockchain technology was soon perceived as disruptive and with much greater potential than cryptocurrencies. And it has come to be seen in the last ten years as a high-potential technology [1]. The reality, however, has shown that its maturation process is still ongoing.

With. The P2P network has users who share tasks, work or files without blockchain technology, it is possible to have an anonymous, decentralized internet with guaranteed privacy protection. The system consists of two parts: a peer-to-peer (P2P) network and a decentralized database the need for a central server. All participants have equal privileges and influence on the environment [2]. Each computer in the network is a node and, whenever new data enters the system, all nodes receive it. This information is encrypted and there is no way to track who added it, it is only possible to verify its validity. As a security measure, the method makes the distributed registration of information to decentralize the process [3]. Thus, when one node leaves the network, the others already have a copy of all the information shared. Likewise, if new nodes enter it, the rest create copies of your information for them.

According to the Gartner Group's 2019 CIO Agenda survey, 60\% of CIOs said they expected some level of adoption of blockchain technologies in the subsequent three years [2]. This contrasts with the scenario of the same survey conducted in 2018, the result of which indicated that only $22 \%$ of CIOs shared this expectation at the time. After a growing interest in 2018 [3], and the appearance of more applications in different sectors in 2019, the forecast was that 2020 would be the year of the blockchain to take off. 


\section{E-Government as a New Paradigm of Public Administration}

In the 1990s, there was great enthusiasm for the development of electronic government or e-Government [4], which was driven by the globalization of the economy, the proliferation of the internet, but also due to a new attitude of the political class towards the use of Information Technologies (IT) in Public Administration (PA).

Over the past few decades, the attention and interest of political power in egovernment has evolved, with a widespread recognition today of the importance of IT as an instrument for the transformation and modernization of PA.

In addition to efficiency gains in terms of the internal functioning of the administrative machine, the use of IT has been seen as an opportunity to change the paradigm of a PA centered on the agency, to one more centered on the citizen and companies, capable of providing quality services and information, accessible at any time, and according to the needs of those looking for them.

E-Government consists of the use of information technologies to deliver state products and services to all citizens, and to industry. This type of tool brings the Government closer to all citizens of a given country [5].

The development strategies for e-government are based on three essential aspects (Fig. 1): user-centricity (the focus on the citizen with the intention of facilitating and globalizing citizens' access to public services); transparency and accountability (in order to promote effective public management, the results of which are measurable and more easily scrutinized); and e-participation (consultation or participation of citizens, as a process of co-creating new policies, services or projects).

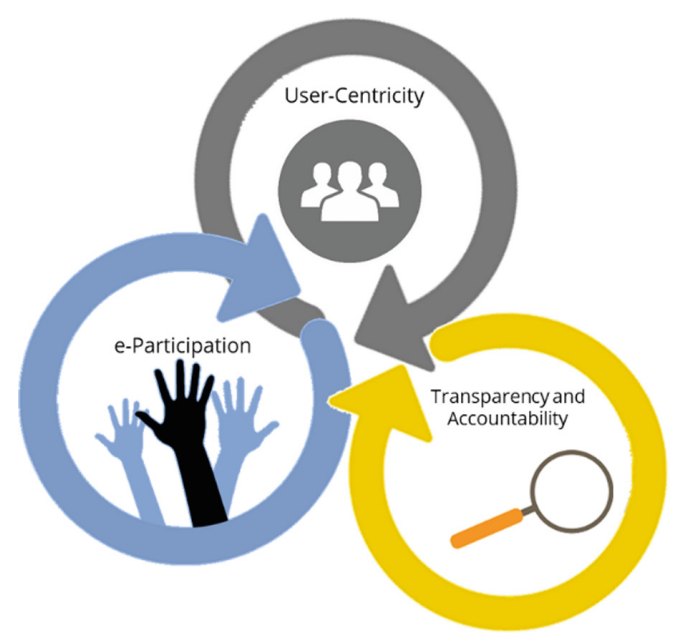

Fig. 1. Basis of development strategies for e-government. 
Civil society, increasingly demanding in terms of quality, accessibility, efficiency and transparency in the services provided by the State. The strategic challenge for PA is, therefore, to provide electronic services of high quality and usability, in order to match the level offered by some services in the private sector.

However, improving the services provided to citizens and businesses depends, first of all, on the optimization of internal processes, the reduction of time in the execution of tasks, and greater cooperation between public bodies, which requires the ability to interoperate both between information systems, either between sectors and government organizations.

The realization of e-government and a transparent, effective and rigorous PA is only possible with the use of solid tools and technologies, capable not only of ensuring the security of communications and data privacy, but also of responding to the specificities and requirements of operation various public bodies.

\section{Blockchain Technologies}

Blockchain emerged as a Bitcoin system, mainly after the publication of the Bitcoin article "A Peer-to-Peer Electronic Cash System". The author was famous for his pseudonym Satoshi Nakamoto [1, 6].

Blockchain terminology refers to a chain of blocks, a series of blocks of data that are chained together cryptographically.

Blockchain came to be known as the system that would conduct the transactions of all Bitcoins and later other cryptocurrencies [7]. The system has come to be widely used by security for those who choose this type of currency in the digital environment [8].

The blockchain is a network that works with very secure chained blocks that always carry content along with a fingerprint. In the case of bitcoin, this content is a financial transaction. The catch here is that the back block will contain the fingerprint of the previous one plus its own content and, with these two pieces of information, generate your own fingerprint [9]. And so on.

Blockchain is not a new technology. It is a combination of proven technologies and applied in a new way (internet, private key encryption and protocol). The result is a system for digital transactions that does not need a third party to intermediate them.

This technology has the potential to change the way we buy and sell, interact with the Government and verify the authenticity of everything. Blockchain technology combines the openness of the Internet with the security of cryptography to offer everyone a faster and more secure way to verify important information and establish trust [10].

Blockchain networks can differentiate into public or private networks (Fig. 2). The public ones have their own rules, functioning independently of legal or regulatory aspects (the case of Bitcoin), the validators of the transactions are anonymous and the entrance to participate in the network of miners is of free access. Private bockchain networks follow regulations, and participants are pre-selected, applications are restricted to closed corporations [11]. That is, the encrypted access keys for carrying out operations on an open network are widely accessible and anonymous, while on a private network, access keys are controlled and there is a need to request permission for transactions [12]. 


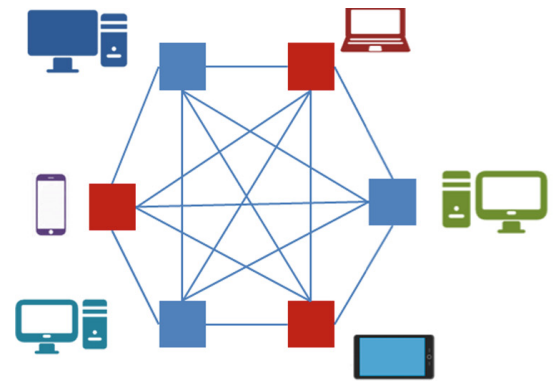

a) Private Network

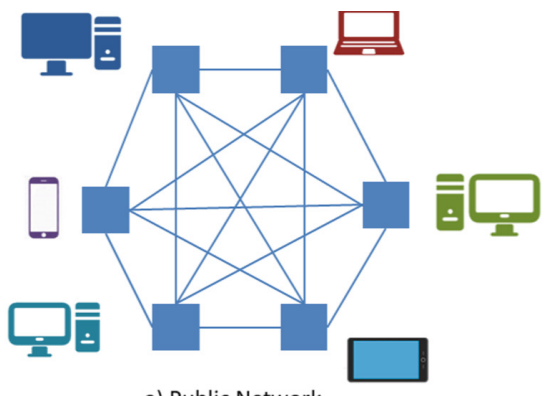

a) Public Network

Fig. 2. Blockchain networks: Validator node: validates, initiates or receives a transaction; Member node: only initiates or receives a transaction.

The main innovative feature of blockchain technology, is the ability to track transactions in decentralized and public databases, reducing the possibility of counterfeiting and fraud [13]. The distributed ledger provides an almost immutable record and guarantees the traceability of transactions since it will be very difficult to manipulate the data on the blockchain, since the changes are immediately reflected in all copies of the reason by the network and they are linked to the previous transaction [10].

An increasing number of countries are investing in applications of blockchain technology, aiming to improve the provision of public services and improve the governance of their countries [14].

Blockchain can add value to public administration due to its properties of immutability, transparency, traceability, reliability and operational resilience.

\section{The Benefits and Applications of Blockchain for Governments}

Governments have the task of facilitating transparency, especially in governance, in the distribution of resources and in achieving greater efficiency, among other things. For these reasons, the governments of many countries have expressed an interest in technology.

Blockchain orders for governments can be the missing link in helping governments become fully digitized. The world has been on the path of digitization, as has been seen in many sectors, such as retail and entertainment, among others. Governments have also felt pressure to follow suit, but it is easier said than done. One of the biggest obstacles that have stood in the way of digitization for many governments is the issue of security.

Bringing personal data of millions of people to digital platforms represents a great risk in case the system is hacked. However, blockchain has been touted as safe due to its structure and that means it could offer a viable solution that can help governments finally go digital. The fact that blockchain is virtually non-hackable makes it more attractive for the development of digital systems for governments. However, this is just 
the tip of the proverbial iceberg compared to what blockchain-based government services can achieve [15].

Blockchain technology offers new opportunities for Governments, different levels: transparency and access to information; control against fraud; the highest quality of public data, control and information security; greater efficiency; and greater confidence in public administration [16].

Blockchain technology can be used successfully by governments in several areas, such as taxes, payments, citizens' digital IDs, legal applications, security, online protection, health services, among many others (Fig. 3).

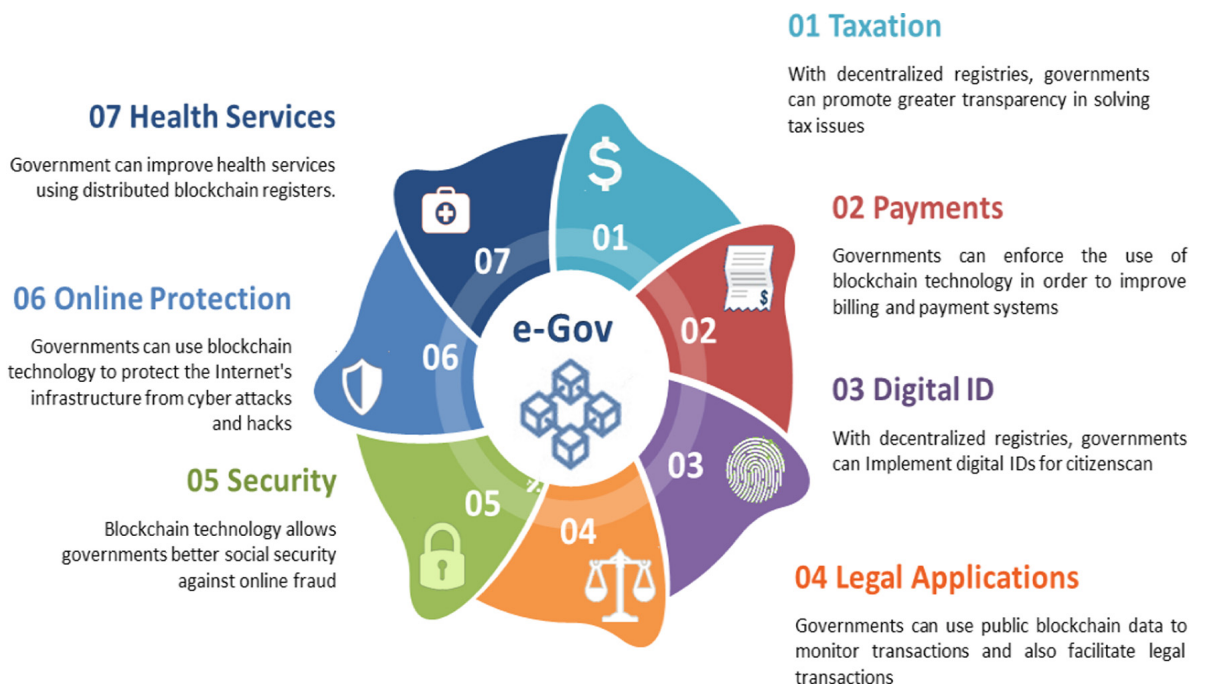

Fig. 3. Government blockchain and its applications.

Taxation, especially regarding the use of digital currencies to avoid taxation, can be controlled through the use of public blockchains and non-private currencies, and can also be the solution for fixing double taxation. In this sense, governments can use blockchain technology to implement protocols that can be used to reduce VAT deficits and reduce the tax burden, eliminating double taxation $[17,18]$.

The creation of online digital IDs to carry out public services in the self-service modality, will allow a greater number of citizens to have access to services that require the use of digital certificates [19].

Governments can use data from public blockchains to track financial transactions in a similar way to how fiat currency transactions are monitored to ensure that the system does not facilitate illegal transactions. The implementation of this technology for a government can be a fundamental tool to ensure that financial transactions in the digital domain remain legal. 
The blockchain decentralizes data and stores it more securely. Thus, it makes it possible to track information and cybersecurity is one of the main results [20]. Blockchain distributed registration technology, due to its secure and immutable character, is ideal to meet the new requirements of governments and function as a reliable repository for identification purposes, and could be the answer to the fight against money laundering and enterprise fraud.

Blockchain applications in the public sector can help governments ensure better protection over their critical infrastructure, thus keeping cyberattacks at a distance. Most of the critical systems used by governments around the world to facilitate service delivery are connected to the Internet. This emphasizes the importance of security for critical systems and like those that cannot be hacked, then it is potentially the best solution. A decentralized registry can also be developed in such a way that it is able to track the integrity of government systems. This would significantly reduce the chances of attacks and data tampering.

Governments can leverage blockchain technology to bring significant improvements to healthcare sectors by developing healthcare systems that offer more efficiency when handling and storing medical data.

\section{Conclusions}

Blockchain technology has been referred to as one of the most today transformative technologies, being one of the most obvious use cases the application of cryptocurrencies. It is the same technology on which cryptocurrencies are based, but they have many other applications. Blockchain can be described as a highly secure and decentralized accounting system in which information can be stored, but cannot be changed. Instead of storing data on servers as used to through cloud storage, blockchain focuses on using a network of computers that store and verify data. Computers on a given network can be distributed worldwide and the network is not centrally controlled.

Governments are generally criticized for not knowing how to handle most of their processes efficiently, but new technologies, such as blockchain, have the potential to bring about drastic changes in the situation through blockchain solutions for the entire government. Governmental Blockchain may be something that will become a reality in the coming years, as governments begin to adopt this technology.

Blockchain technology can increase access and transparency of information, the predictive capacity of data, control of public data, control against corruption and fraud, and information security [21], enabling the provision of more efficient public services by improving business processes for government actors at any level of government; and enable the creation of fast, cheap and especially safe public records.

Blockchain can be very beneficial for public institutions, making them more transparent, close and secure. However, administrations also have to reposition themselves in this new scenario, since it is not only about its benefit, but also the commitment to promote this technology that will have an impact on the entire economy. These benefits can contribute to the observance of governance principles and, consequently, contribute to good governance in the public sector. 


\section{References}

1. Nakamoto, S.: Bitcoin: a peer-to-peer electronic cash system, Manubot (2019)

2. Zhang, C., Wu, J., Long, C., Cheng, M.: Review of existing peer-to-peer energy trading projects. Energy Procedia 105, 2563-2568 (2017)

3. Puthal, D., Malik, N., Mohanty, S.P., Kougianos, E., Das, G.: Everything you wanted to know about the blockchain: its promise, components, processes, and problems. IEEE Consum. Electron. Mag. 7(4), 6-14 (2018)

4. Gartner: Gartner Group's 2019 CIO Agenda survey, Gartner (2019)

5. Gartner: Gartner Group's 2018 CIO Agenda survey, Gartner (2018)

6. Guijarro, L.: Analysis of the interoperability frameworks in e-government initiatives. In: International Conference on Electronic Government, Berlin (2004)

7. Twizeyimana, J.D., Andersson, A.: The public value of e-government-a literature review. Gov. Inf. Q. 36(2), 167-178 (2019)

8. Barber, S., Boyen, X., Shi, E., Uzun, E.: Bitter to better-how to make bitcoin a better currency. In: International Conference on Financial Cryptography and Data Security, Berlin (2012)

9. Filippi, P., Loveluck, B.: The invisible politics of bitcoin: governance crisis of a decentralized infrastructure. Internet Policy Rev. 5(4), 1-32 (2016)

10. Abeyratne, S.A., Monfared, R.P.: Blockchain ready manufacturing supply chain using distributed ledger. Int. J. Res. Eng. Technol. 5(9), 1-10 (2016)

11. Swan, M.: Blockchain: Blueprint for a New Economy. O'Reilly Media, Inc., Sebastopol (2015)

12. Natarajan, H., Krause, S., Gradstein, H.: Distributed Ledger Technology and Blockchain. World Bank, New York (2017)

13. Li, X., Jiang, P., Chen, T., Luo, X., Wen, Q.: A survey on the security of blockchain systems. Future Gene. Comput. Syst. 107, 841-853 (2020)

14. Reijers, W., Coeckelbergh, M.: The blockchain as a narrative technology: Investigating the social ontology and normative configurations of cryptocurrencies. Philos. Technol. 31(1), 103-130 (2018)

15. Jun, M.: Blockchain government-a next form of infrastructure for the twenty-first century. J. Open Innov. Technol. Market. Complex. 4(7) 1-12 (2018)

16. Boireau, O.: Securing the blockchain against hackers. Network Secur. 2018(1), 8-11 (2018)

17. Saberi, S., Kouhizadeh, M., Sarkis, J., Shen, L.: Blockchain technology and its relationships to sustainable supply chain management. Int. J. Prod. Res. 57(7), 2117-2135 (2019)

18. Tasatanattakool, P., Techapanupreeda, C.: Blockchain: challenges and applications. In: International Conference on Information Networking (ICOIN) (2018)

19. Fulmer, N.: Exploring the legal issues of blockchain applications. Akron L. Rev. 52(1), 162187 (2019)

20. Alexopoulos, C., Charalabidis, Y., Androutsopoulou, A., Loutsaris, M.A., Lachana, Z.: Benefits and obstacles of blockchain applications in E-government. In: 52nd Hawaii International Conference on System Sciences (2019)

21. Zyskind, G., Nathan, O.: Decentralizing privacy: using blockchain to protect personal data. In: IEEE Security and Privacy Workshops (2015)

22. Per, A.: Blockchain as an anti-corruption tool: case examples and introduction to the technology. U4 Anticorruption Tool 2020(7), 1-33 (2020) 